\title{
Efficacy and Safety of Antipsychotics for the Treatment of Major Depressive Disorder in Adolescents and Adults: Current Issues and Clinical Perspective
}

\author{
Raj Rasasingham ${ }^{1,2}$ \\ ${ }^{1}$ University of Toronto/Humber Hospital, Toronto, Canada \\ ${ }^{2}$ Harvard University/Children's Hospital, Boston, USA \\ Email: rajrasas@hotmail.com
}

Received 1 May 2014; revised 30 May 2014; accepted 25 June 2014

Copyright (C) 2014 by author and Scientific Research Publishing Inc. This work is licensed under the Creative Commons Attribution International License (CC BY). http://creativecommons.org/licenses/by/4.0/

(c) (i) Open Access

\section{Abstract}

Objectives: Atypical antipsychotics are increasingly being prescribed for the treatment of mood disorders, often off-label. This review describes existing published literature on the efficacy and safety of antipsychotics for the treatment of major depressive disorder (MDD) in adults and adolescents. We discuss current clinical considerations: role as monotherapy, adjunctive, or augmentation therapy, side effect profiles, and optimal dose and duration of therapy. Potential areas for future research and methodological considerations are also highlighted. Method: We conducted a literature search of MEDLINE, EMBASE, PsycINFO, and Cochrane databases. Relevant articles and references were identified. MDD practice guidelines for adolescents and adults were reviewed. Results: Evidence for using atypical antipsychotics to treat MDD is heterogeneous. It is thus difficult to draw firm conclusions regarding their role in therapy. Most current guidelines do not include recommendations for atypical antipsychotics, but off-label use is common in clinical practice. Primary use is adjunct or augmentation therapy for treatment resistant depression. Potential benefits versus side effects must be cautiously considered, especially for children and adolescents. Clinicians must rely on their clinical experience and professional judgment to determine an optimal dose and duration of therapy. Conclusions: There is progressive research to support the use of atypical antipsychotics to treat MDD in adults. However, additional research and well-designed studies are needed to determine the appropriate and safe use of atypical antipsychotics for treating child and adolescent depression. Other subpopulations that may benefit from combination therapy, such as individuals with dual diagnoses, may be identified through future research. 


\section{Keywords}

\section{Atypical Antipsychotics, Major Depressive Disorder, Depression}

\section{Introduction}

The clinical role for atypical antipsychotics has expanded and received much attention in recent years. Typically indicated for schizophrenia and bipolar disorder, antipsychotics are increasingly being used off-label for the treatment of generalized anxiety and mood disorders. Antipsychotics are a recent addition to available treatments for adult major depressive disorder (MDD). Quetiapine fumarate extended release (ER), a second-generation/atypical antipsychotic (SGA), is currently the only antipsychotic with an approved indication for MDD in Canada. In 2009, the Food and Drug Administration (FDA) approved quetiapine and quetiapine ER as monotherapy in bipolar depression and quetiapine ER as augmentation for MDD. Aripiprazole, another atypical antipsychotic, is also indicated as augmentation for MDD. The combination olanzapine/fluoxetine is approved for MDD as well as bipolar depression.

Major Depression or Clinical Depression involves 5 key symptoms for a two week period. At least one of those symptoms must be depressed mood or loss of interest or pleasure. The other symptoms revolved around weight changes, sleep changes, fatigue or loss of energy for nearly the whole day, psychomotor retardation or agitation, feeling of guilt/worthlessness, diminished ability to concentrate and thoughts of dying.

Current guidelines for the management of MDD recommend selective serotonin receptor inhibitors (SSRIs) such as citalopram, escitalopram, fluoxetine, paroxetine, and sertraline, or serotonin-norepinephrine receptor inhibitors (SNRIs) such as duloxetine, venlafaxine, and desvenlafaxine, as first-line therapy [1]. First-generation tricyclic and monoamine oxidase inhibitor antidepressants are generally considered second- or third-line treatments due to significant side effects and drug interactions. Quetiapine is currently listed as a second-line therapy [1]. We have also considered therapy as modality of treatment of depression. Cognitive Behavioral Therapy and Interpersonal Therapy have also shown benefits in the treatment of depression. Electroconvulsive Therapy has shown benefit in treatment resistant depression. However, the focus of this paper is antipsychotics as an option for treatment resistant depression.

Selection of antidepressant therapy remains dependent on variable patient response to the medication. Evidence on the efficacy and tolerability differences between newer agents may be used as a guide for selection of initial therapy. Initial antidepressant therapy is typically given an adequate trial period of 4 - 6 weeks to determine if the patient is responsive to the medication. However, response to therapy varies between patients. Some individuals may feel improvement within a few days, while others may not experience significant benefit for weeks. There is inconclusive evidence to suggest switching within a class of medication yields better results compared to switching between classes of medications, or vice versa [1]. Guidelines generally recommend switching within class if the patient is responding to therapy, but experiencing intolerable side effects. Switching to a different class is recommended if a patient is non-responsive after an adequate trial period. Atypical antipsychotics may be used as adjunctive or augmentation therapy when patients exhibit a partial response to therapy, or when patients have treatment-resistant depression [2]-[10]. Use of antipsychotics is also recommended for depression with psychotic features.

SSRIs are among the most commonly used first-line agents for the treatment of MDD. SSRI's efficacy is well documented in literature. However, there have been case reports of potential increased suicidal ideation with the use of SSRIs, particularly in the adolescent and young adult populations. Clinicians are therefore considering alternative therapies such as atypical antipsychotics. Although this mitigates the increased risk of suicidal ideation, use of atypical antipsychotics is associated with other significant side effects. Patients should be educated on the potential benefit of improved quality of life versus the risk of side effects due to the medication. In this paper, we review the current literature on the efficacy and safety of using antipsychotics for the treatment of depression in adults and adolescents. We also discuss directions for future research initiatives.

\section{Methods}

We conducted a literature search of the MEDLINE (1946 to November 2012), EMBASE (1980 to 2012), Psy- 
cINFO (1806 to 2012), and Cochrane Library databases. Keyword search terms were variations on: depression, major depressive disorder, treatment-resistant depression, nonresponsive depression, antipsychotics, secondgeneration antipsychotics, generic names of antipsychotics. We restricted literature to English and human subjects. Systematic literature reviews, meta-analyses, randomized controlled trials (RCTs) and case reports were selectively reviewed. Current North American guidelines by the Canadian Network for Mood and Anxiety Treatments (CANMAT), and the American Academy of Child and Adolescent Psychiatry (AACAP) for the treatment of adult and adolescent depression were reviewed [11] [12]. Relevant references from systematic reviews, meta-analyses, and guidelines were identified.

Antipsychotics can help for psychosis management and depression treatment in adolescents. However, close supervision is required to monitor side effects such as somnolence, prolactin elevation, metabolic disturbances and extrapyramidal symptoms.

\section{Evidence for Antipsychotic Efficacy for MDD in Children and Adolescents}

AACAP guidelines recommend antipsychotic therapy in combination with SSRIs for youths with psychotic depression [12]. Antidepressants alone may be suitable for vague or mild psychotic symptoms. It is recommended that antipsychotics be slowly tapered off when psychotic symptoms have improved. The eventual goal is to use monotherapy in adolescents with an antidepressant. The AACAP released guidelines for the use of atypical antipsychotics in children and adolescent in light of the recent FDA approval of their use for the treatment of irritability associated with autistic disorder, schizophrenia, and bipolar I disorder with mixed or manic episodes among individuals less than 18 years of age [13]. Use of these medications specifically for the treatment of MDD in children and adolescents is not discussed. However, antipsychotics are recommended for augmentation in treatment-resistant depression, recurrent depression, and psychotic depression in the NICE guidelines (National Institute for Health and Clinical Excellence. 2005) [14].

Polypharmacy for the treatment of MDD in pediatric patients has been reported to have increased from $7.9 \%$ in 1996 to $45 \%$ in 2005 [15]. A trend towards combining antidepressants with antipsychotics over antidepressants with stimulants was evident. There needs to be stronger empiric evidence for combining SGAs and SSRIs for treatment-resistant depression or the management of residual symptoms to be able to recommend this combination in clinical guidelines [16]. However, combination therapy is used in clinical practice.

Disruptive behavior disorders were found to be the most common diagnosis in physician visits for antipsychotics among children and adolescents from 2005-2009 [17]. Comparatively, depression was found to be the most common diagnosis in physician visits for antipsychotics overall at $21.6 \%$ and the second most common diagnosis without an FDA indication at 10.9\% among adults from 2005-2009. Adults were found to have more medical visits with a prescription for antipsychotics than adolescents or children, but the rate of prescribing among adolescents or children is increasing [17]. Contributing factors to this trend include the availability of formulations that are more convenient to administer to children, and their improved tolerability due to fewer extrapyramidal side effects compared to first generation antipsychotics [18].

\section{Evidence for Antipsychotic Efficacy for MDD in Adults}

Systematic reviews and meta-analyses identified primarily focused on the use of atypical antipsychotics for: offlabel indications [19], treatment-resistant depression, augmentation therapy [20]-[22], or monotherapy [23]. There were also a number of reviews that summarize current evidence [24]. A 2010 Cochrane systematic review on the effects and safety of atypical antipsychotics in the treatment of MDD and dysthymia identified 28 randomized, double-blind trials comparing five oral atypical antipsychotics: amisulpride, aripiprazole, olanzapine, quetiapine, and risperidone, alone or for augmentation with other pharmacotherapy or placebo [25]. It appeared that only quetiapine indicated benefit alone or as additional treatment compared to placebo for the treatment of MDD. One meta-analysis found combination antidepressant with antipsychotic was superior to monotherapy with either antidepressant or antipsychotic in the acute treatment of psychotic depression [23]. Augmentation with atypical antipsychotics has also been shown to improve remission or clinical response compared to placebo [21] [22]. The number of previous episodes of depression and the presence of psychotic symptoms has been shown to be associated with the adoption of augmentation therapy with a non-antidepressant agent such as an atypical antipsychotic [26]. 


\section{Optimal Dosing and Duration of Therapy}

Similar to many other drugs, atypical antipsychotics are often used off-label for pediatric populations. Dosing is established in adults, and limited data exists to guide clinicians in determining the appropriate and safe dose and duration of therapy in younger patients. General principles are to titrate doses up slowly to mitigate the risk of side effects [13]. Extrapyramidal symptoms, sedation, and weight gain are of particular concern. These adverse effects may interfere with adherence to the medication. Research is needed to determine the future health repercussions of such side effects with extended duration of therapy in young patients [13]. When used in combination with, for example, an antidepressant, lower doses of each medication may be sufficient to achieve the desired therapeutic effect. Clinical experience and extrapolation from existing literature on the use of atypical antipsychotics for the treatment of schizophrenia and ADHD in younger populations should be used to guide treatment of MDD in children and adolescents.

\section{Side Effects and Monitoring}

There have been reports and evidence of increased mood lability and suicidal ideation with the use of SSRIs in both adolescents and adults. Antipsychotics are being studied to determine their potential as an alternative therapy, and are used off-label in clinical practice. However, they are also associated with significant adverse effects that should be taken into consideration. A recent meta-analysis on the adverse effects of atypical antipsychotics in children and adolescents found an increased risk of somnolence/sedation, extrapyramidal syndrome, and metabolic syndrome [27]. Another study examined the differences in tolerability as determined by discontinuation of atypical antipsychotics due to adverse events in the treatment of bipolar depression, MDD, and generalized anxiety disorder [28]. Commonly reported adverse events were somnolence, sedation, weight again, and akathisia. Other common side effects of atypical antipsychotics are dyslipidemia and hyperglycemia.

There is limited evidence of ethnic differences in the risk of adverse events to antipyschotics in the treatment of psychoses and depression [29]. There may also be subtle differences between atypical antipsychotics. For example, one study found that aripiprazole was independently associated with better health-related quality of life and health utilities compared to other atypical antipsychotics in the treatment of depression [30]. Overall, the risk of side effects must be weighed against the benefit of improved function and quality of life. Therapy should be optimized to minimize side effects and maximize benefits according to the individual patient response to the medication. Baseline and ongoing monitoring of metabolic side effects and extrapyramidal symptoms should be performed. Diagnoses from adolescence to adulthood are variable and not static. Clinicians should reassess a patient's original diagnoses as symptoms develop or regress, and adjust therapy accordingly.

\section{Potential Areas for Future Research}

The Agency for Healthcare Research and Quality (AHRQ) identified several areas for future research on the use of first- and second-generation antipsychotics in children and young adults [31]. Selecting therapy for psychiatric conditions is inherently difficult given the heterogeneity of evidence and variable patient response to available therapies. Heterogeneity of currently available evidence limits comparison between trials. Standardized measures of assessing the patient's clinical status, response to therapy, and adverse events experienced may allow for greater knowledge of the patient populations who would benefit from treatment with atypical antipsychotics. Assessment tools such as the Hamilton Rating Scale for Depression (HAM-D) and the MontgomeryÅsberg Depression Rating Scale (MADRS) are the most widely used in clinical practice. Clinicians must rely on clinical experience and judgment to guide their treatment decisions.

Further research is needed to determine subgroups of populations (e.g. age, race/ethnicity, co-morbidities, and treatment failure) that would benefit from atypical antipsychotics, and the appropriate dosing and duration of treatment for clinical benefit. A correlation between mood disorders and substance use disorders is well-documented [32]. Although evidence for treating substance abuse with antipsychotics is not compelling, there is some evidence examining the benefit of antipsychotics for dual diagnosis with mood disorders. Quetiapine has been shown to improve psychiatric symptoms and cocaine cravings in patients with bipolar disorder and cocaine dependence [33]. However, there is no literature to our knowledge that specifically examines the role of antipsychotics in MDD comorbid with substance abuse. Evidence for using antipsychotics in dual diagnosis patients is primarily in the area of schizophrenia or bipolar disorder and substance use disorders. A study identified sev- 
en articles examining dual diagnosis psychotic disorders and substance abuse in pediatric populations [34]. Clozapine, olanzapine, and aripiprazole are potentially beneficial in this population. Given the potential role of antipsychotics for other dual diagnoses that often have a mood disorder component as well as the indication for quetiapine as monotherapy for MDD, it is reasonable to extrapolate a potential benefit for patients with dual diagnoses with MDD. More research is needed to better understand the role of antipsychotics for the treatment of dual diagnoses. Polypharmacy involving antidepressants and antipsychotics for the treatment of MDD in children and adolescent patients is another area that is understudied [16].

\section{Conclusion}

In this review, we discussed current issues surrounding the use of antipsychotics for major depressive disorder in adolescents and adults. We also highlight gaps in the literature on the use of antipsychotics for major depressive disorder. Clinicians and researchers continue to search for novel applications/indications for atypical antipsychotics. Evidence of efficacy as augmentation for MDD patients who have not responded adequately to selective serotonin reuptake inhibitors/serotonin-norepinephrine reuptake inhibitors (SSRIs/SNRIs) has increased over the past decade. Antipsychotics may be considered as adjunctive or augmentation therapy for patients with treatment-resistant depression. Further research is needed to study the efficacy and safety of atypical antipsychotics in subset populations such as adolescents or substance abuse.

\section{Disclosure}

Author state has no conflicts of interest.

\section{References}

[1] Lam, R.W., et al. (2009) Canadian Network for Mood and Anxiety Treatments (CANMAT) Clinical Guidelines for the Management of Major Depressive Disorder in Adults. III. Pharmacotherapy. Journal of Affective Disorders, 117, S26S43. http://dx.doi.org/10.1016/j.jad.2009.06.041

[2] Corya, S.A., et al. (2006) A Randomized, Double-Blind Comparison of Olanzapine/Fluoxetine Combination, Olanzapine, Fluoxetine, and Venlafaxine in Treatment-Resistant Depression. Depression and Anxiety, 23, 364-372. http://dx.doi.org/10.1002/da.20130

[3] Shelton, R.C., Williamson, D.J., Corya, S.A., Sanger, T.M., Van Campen, L.E., Case, M., Briggs, S.D. and Tollefson, G.D. (2005) Olanzapine/Fluoxetine Combination for Treatment-Resistant Depression: A Controlled Study of SSRI and Nortriptyline Resistance. The Journal of clinical psychiatry, 66, 1289-1297. http://dx.doi.org/10.4088/JCP.v66n1012

[4] Thase, M.E., Corya, S.A., Osuntokun, O., Case, M., Henley, D.B., Sanger, T.M., Watson, S.B. and Dubé, S. (2007) A Randomized, Double-Blind Comparison of Olanzapine/Fluoxetine Combination, Olanzapine, and Fluoxetine in Treatment-Resistant Major Depressive Disorder. The Journal of Clinical Psychiatry, 68, 224-236. http://dx.doi.org/10.4088/JCP.v68n0207

[5] McIntyre, A. and Gendron, A. (2007) Selective Serotonin Reuptake Inhibitors or Venlafaxine in Patients with Major Depression, Comorbid Anxiety, and Residual Depressive Symptoms: A Randomized, Placebo. Depression and Anxiety, 494, 487-494.

[6] Bauer, M., Pretorius, H.W., Constant, E.L., Earley, W.R., Szamosi, J. and Brecher, M. (2009) Extended-Release Quetiapine as Adjunct to an Antidepressant in Patients with Major Depressive Disorder: Results of a Randomized, Placebo-Controlled, Double-Blind Study. The Journal of Clinical Psychiatry, 70, 540-549. http://dx.doi.org/10.4088/JCP.08m04629

[7] El-Khalili, N., Joyce, M., Atkinson, S., Buynak, R.J., Datto, C., Lindgren, P. and Eriksson, H. (2010) Extended-Release Quetiapine Fumarate (Quetiapine XR) as Adjunctive Therapy in Major Depressive Disorder (MDD) in Patients with an Inadequate Response to Ongoing Antidepressant Treatment: A Multicentre, Randomized, Double-Blind, Placebo-Controlled Study. The International Journal of Neuropsychopharmacology, 13, 917-932. http://dx.doi.org/10.1017/S1461145710000015

[8] Rapaport, M.H., et al. (2006) Effects of Risperidone Augmentation in Patients with Treatment-Resistant Depression: Results of Open-Label Treatment Followed by Double-Blind Continuation. Neuropsychopharmacology, 31, 2505-2513. http://dx.doi.org/10.1038/sj.npp.1301113

[9] Keitner, G.I., Garlow, S.J., Ryan, C.E., Ninan, P.T., Solomon, D.A., Nemeroff, C.B. and Keller, M.B. (2009) A Randomized, Placebo-Controlled Trial of Risperidone Augmentation for Patients with Difficult-to-Treat Unipolar, NonPsychotic Major Depression. Journal of Psychiatric Research, 43, 205-214. 
http://dx.doi.org/10.1016/j.jpsychires.2008.05.003

[10] Mahmoud, R., Pandina, G.J., Turkoz, I., Kosik-Gonzalez, C., Canuso, C.M., Kujawa, M.J. and Gharabawi-Garibaldi, G.M. (2007) Risperidone for Treatment-Refractory Major Depressive Disorder. Annals of Internal Medicine, 147, 593-602. http://dx.doi.org/10.7326/0003-4819-147-9-200711060-00003

[11] Kennedy, S.H., Lam, R.W., Parikh, S.V., Patten, S.B. and Ravindran, A.V. (2009) Canadian Network for Mood and Anxiety Treatments (CANMAT) Clinical Guidelines for the Management of Major Depressive Disorder in Adults. Introduction. Journal of Affective Disorders, 117, S1-S2. http://dx.doi.org/10.1016/j.jad.2009.06.043

[12] Birmaher, B. and Brent, D. (2007) Practice Parameter for the Assessment and treatment of Children and Adolescents with Depressive Disorders. Journal of the American Academy of Child and Adolescent Psychiatry, 46, 1503-1526. http://dx.doi.org/10.1097/chi.0b013e318145ae1c

[13] Findling, R.L., et al. (2011) Practice Parameter for the Use of Atypical Antipsychotic Medications in Children and Adolescents. Journal of the American Academy of Child and Adolescent Psychiatry.

[14] National Institute for Health and Clinical Excellence (2005) Depression in Children and Young People. CG28 (September).

[15] McIntyre, R.S. and Jerrell, J.M. (2009) Polypharmacy in Children and Adolescents Treated for Major Depressive Disorder: A Claims Database Study. The Journal of Clinical Psychiatry, 70, 240-246.

http://www.ncbi.nlm.nih.gov/pubmed/19192465 http://dx.doi.org/10.4088/JCP.08m04212

[16] Díaz-Caneja, C.M., et al. (2012) Polypharmacy with Antidepressants in Children and Adolescents. The International Journal of Neuropsychopharmacology, 17, 1063-1082.

[17] Olfson, M., Blanco, C., Liu, S.M., Wang, S. and Correll, C.U. (2012) National Trends in the Office-Based Treatment of Children, Adolescents, and Adults with Antipsychotics. Archives of General Psychiatry, 69, 1247-1256.

http://www.ncbi.nlm.nih.gov/pubmed/22868273 http://dx.doi.org/10.1001/archgenpsychiatry.2012.647

[18] Vitiello, B., Correll, C., van Zwieten-Boot, B., Zuddas, A., Parellada, M. and Arango, C. (2009) Antipsychotics in Children and Adolescents: Increasing Use, Evidence for Efficacy and Safety Concerns. European Neuropsychopharmacology, 19, 629-635. http://www.ncbi.nlm.nih.gov/pubmed/19467582 http://dx.doi.org/10.1016/j.euroneuro.2009.04.008

[19] Fountoulakis, K. and Nimatoudis, I. (2004) Off-Label Indications for Atypical Antipsychotics: A Systematic Review. Annals of General Hospital Psychiatry, 3, 4.

[20] Fleurence, R., Williamson, R., Jing, Y., Kim, E., Tran, Q.V., Pikalov, A.S. and Thase, M.E. (2009) A Systematic Review of Augmentation Strategies for Patients with Major Depressive Disorder. Psychopharmacology Bulletin, 42, 57 90.

[21] Papakostas, G.I., Shelton, R.C., Smith, J. and Fava, M. (2007) Augmentation of Antidepressants with Atypical Antipsychotic Medications for Treatment-Resistant Major Depressive Disorder: A Meta-Analysis. Journal of Clinical Psychiatry, 68, 826-831. http://dx.doi.org/10.4088/JCP.v68n0602

[22] Nelson, J. and Papakostas, G. (2009) Atypical Antipsychotic Augmentation in Major Depressive Disorder: A Meta-Analysis of Placebo-Controlled Randomized Trials. American Journal of Psychiatry, 166, 980-991. http://dx.doi.org/10.1176/appi.ajp.2009.09030312

[23] Farahani, A. and Correll, C.U. (2012) Are Antipsychotics or Antidepressants Needed for Psychotic Depression? A Systematic Review and Meta-Analysis of Trials Comparing Antidepressant or Antipsychotic Monotherapy with Combination Treatment. The Journal of Clinical Psychiatry, 73, 486-496. http://dx.doi.org/10.4088/JCP.11r07324

[24] Chen, J., Gao, K.M. and Kemp, D.E., (2011) Second-Generation Antipsychotics in Major Depressive Disorder: Update and Clinical Perspective. Current Opinion in Psychiatry, 24, 10-17. http://dx.doi.org/10.1097/YCO.0b013e3283413505

[25] Komossa, K., Depping, A. and Gaudchau, A., (2012) Second-Generation Antipsychotics for Major Depressive Disorder and Dysthymia (Review). Cochrane Database of Systematic Reviews.

[26] Garcia-Toro, M., Medina, E., Galan, J.L., Gonzalez, M.A. and Maurino, J. (2012) Treatment Patterns in Major Depressive Disorder after an Inadequate Response to First-Line Antidepressant Treatment. BMC Psychiatry, 12, 143. http://www.pubmedcentral.nih.gov/articlerender.fcgi?artid=3514293\&tool=pmcentrez\&rendertype=abstrac $\mathrm{t}$

http://dx.doi.org/10.1186/1471-244X-12-143

[27] Cohen, D., Bonnot, O., Bodeau, N., Consoli, A. and Laurent, C. (2012) Adverse Effects of Second-Generation Antipsychotics in Children and Adolescents: A Bayesian Meta-Analysis. Journal of Clinical Psychopharmacology, 32, 
309-316. http://dx.doi.org/10.1097/JCP.0b013e3182549259

[28] Gao, K.M., et al. (2011) Number Needed to Treat to Harm for Discontinuation Due to Adverse Events in the Treatment of Bipolar Depression, Major Depressive Disorder, and Generalized. The Journal of Clinical Psychiatry, 72, 10631071. http://dx.doi.org/10.4088/JCP.09r05535gre

[29] Ormerod, S., McDowell, S.E., Coleman, J.J. and Ferner, R.E. (2008) Ethnic Differences in the Risks of Adverse Reactions to Drugs Used in the Treatment of Psychoses and Depression. Drug Safety, 31, 597-607. http://dx.doi.org/10.2165/00002018-200831070-00005

[30] Kalsekar, I., Wagner, J.-S., DiBonaventura, M., Bates, J., Forbes, R. and Hebden, T. (2012) Comparison of HealthRelated Quality of Life among Patients Using Atypical Antipsychotics for Treatment of Depression: Results from the National Health and Wellness Survey. Health and Quality of Life Outcomes, 10, 81. http://dx.doi.org/10.1186/1477-7525-10-81

[31] Christian, R., et al. (2012) Future Research Needs for First- and Second-Generation Antipsychotics for Children and Young Adults. Agency for Healthcare Research and Quality, Rockville.

[32] Lev-Ran, S., Aviram, A., Braw, Y., Nitzan, U., Ratzoni, G. and Fennig, S. (2012) Clinical Correlates of Cannabis Use among Adolescent Psychiatric Inpatients. European Psychiatry, 27, 470-475. http://dx.doi.org/10.1016/j.eurpsy.2011.11.002

[33] Brown, E.S., Nejtek, V.A., Perantie, D.C. and Bobadilla, L. (2002) Quetiapine in Bipolar Disorder and Cocaine Dependence. Bipolar Disorders, 4, 406-411. http://dx.doi.org/10.1034/j.1399-5618.2002.02229.x

[34] Price, S.A. and Brahm, N.C. (2011) Antipsychotic Treatment of Adolescent Dual Diagnosis Patients. The Journal of Pediatric Pharmacology and Therapeutics, 16, 226-236. 
Scientific Research Publishing (SCIRP) is one of the largest Open Access journal publishers. It is currently publishing more than 200 open access, online, peer-reviewed journals covering a wide range of academic disciplines. SCIRP serves the worldwide academic communities and contributes to the progress and application of science with its publication.

Other selected journals from SCIRP are listed as below. Submit your manuscript to us via either submit@scirp.org or Online Submission Portal.
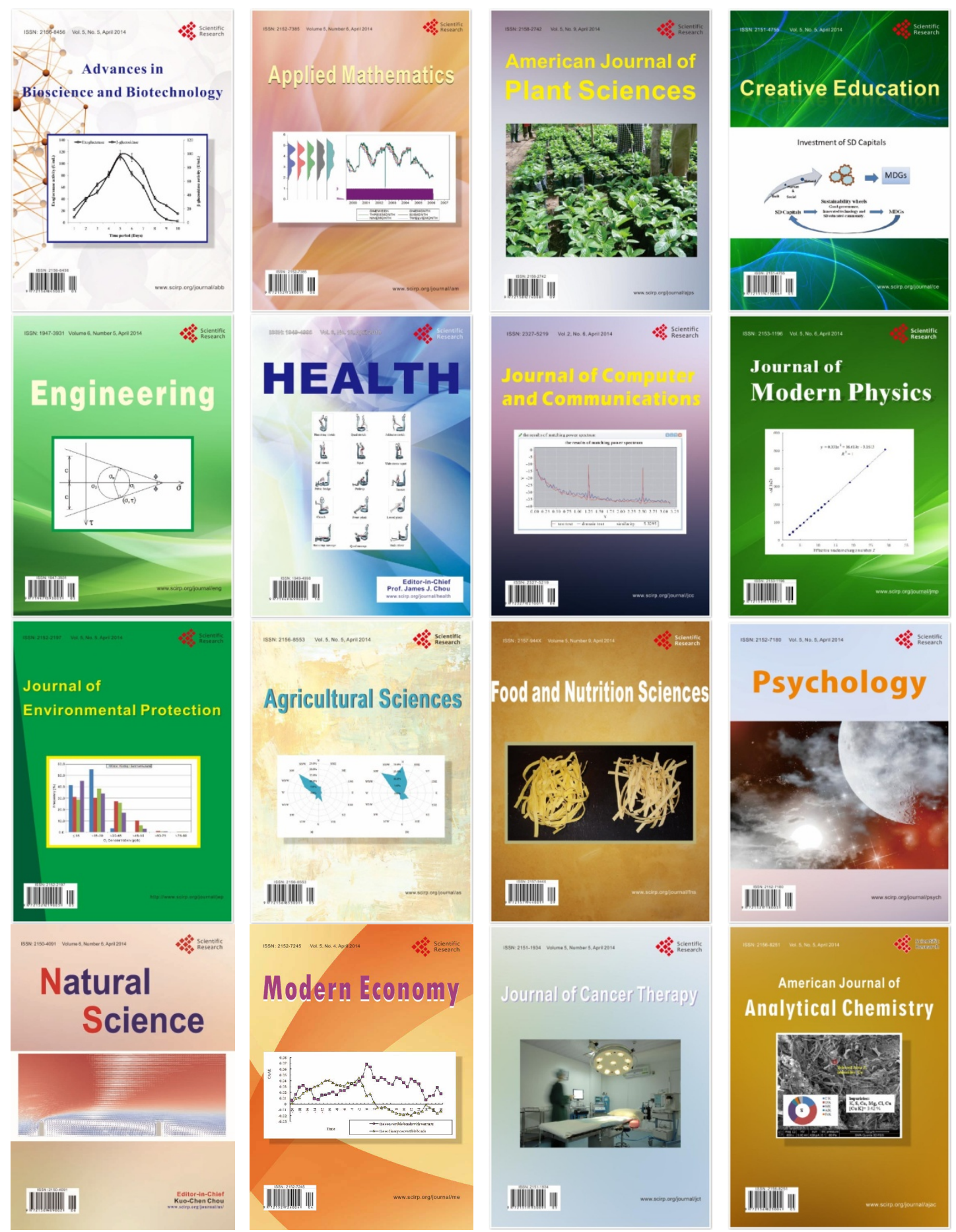